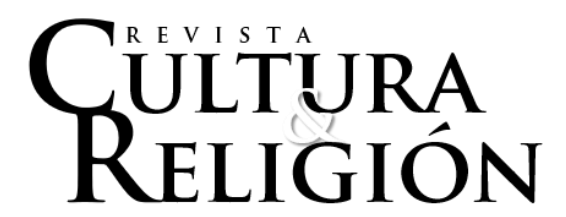

Vol. XIV, N 1 (2020) pp. 100-120

Recibido: 17 de enero, 2020

Aceptado: 1 de abril, 2020

\title{
INCIDENCIA RELIGIOSA EN CLAVE MULTILATERAL: LA PRESENCIA DE REDES POLÍTICAS EVANGÉLICAS EN LAS ASAMBLEAS DE LA OEA*
}

\author{
Religious advocacy in a multilateral context: the presence of evangelical \\ political networks in the assemblies of the OAS
}

\author{
Nicolás Panotto** \\ Universidad Arturo Prat / GEMRIP, Chile \\ nicolaspanotto@gmail.com
}

\begin{abstract}
Resumen
Este artículo propone un análisis sobre los nuevos mecanismos de incidencia del campo evangélico latinoamericano desde un encuadre regional, a partir de un estudio de la emergente presencia de redes políticas y organizaciones evangélicas desde dinámicas transfronterizas dentro del sistema interamericano. Puntualmente, se observa la participación de coaliciones evangélicas en el ámbito del Diálogo con la Sociedad Civil, en el marco de las asambleas anuales y cumbres trianuales de la Organización de Estados Americanos (OEA), a través de un análisis discursivo de las intervenciones de dichas coaliciones sobre el tipo de retórica utilizada y las cosmovisiones político-teológicas de fondo.
\end{abstract}

Palabras clave: redes políticas evangélicas, sistema interamericano, coaliciones, relaciones internacionales, Organización de Estados Americanos.

\begin{abstract}
This article presents an analysis of the new mechanisms of advocacy within the domain of the Latin American evangelical church, and from a regional perspective. It is based on a study of the emerging presence of political networks and evangelical organizations derived from cross-border dynamics within the inter-American system. Specifically, the participation of evangelical coalitions in the area of Dialogue with Civil Society is observed within the framework of the annual assemblies and tri-annual summits of the Organization of American

\footnotetext{
* Los datos utilizados en el presente artículo surgen del trabajo del Grupo de Estudios Multidisciplinarios sobre Religión e Incidencia Pública (GEMRIP) en el proyecto Participación de la Sociedad Civil en la Cumbre de las Américas (PASCA), con el grupo de actores religiosos y espiritualidades del Foro Ciudadano de las Américas.

** Doctor en Ciencias Sociales, Facultad Latinoamericana de Ciencias Sociales, FLACSO (Argentina). Investigador asociado, Instituto de Estudios Internacionales (INTE) de la Universidad Arturo Prat (Chile). https://orcid.org/0000-0002-0513-7175
} 
States (OAS). The study analyzes the coalitions' rhetoric and the underlying politicaltheological worldviews.

Keywords: Evangelical political networks, inter-American system, coalitions, international relations, Organization of American States.

\section{Introducción}

El campo evangélico ha alcanzado un creciente nivel de influencia sociopolítica desde mediados del siglo XX en diversos niveles y desde distintas matrices. Podemos identificar, a modo de distinción analítica, tres instancias principales: incidencia microsocial o comunitaria (enfocada en los procesos de influencia de iglesias evangélicas en espacios sociales concentrados, como pequeños grupos o comunidades, barrios o proyectos de asistencia, y también en el rol de individuos creyentes en la participación de espacios públicos), incidencia a nivel nacional desde un encuadre institucional (focalizada en el intento de conformación de partidos políticos a partir de la década de 1980, la complejización de procesos de lobby a través de partidos en municipios y Estados, y la participación en debates públicos sobre proyectos de ley) y la incipiente incidencia en instancias transnacionales (como en organismos internacionales y proyectos de alcance regional).

El análisis de estas reconfiguraciones se ha vuelto uno de los campos de investigación más fecundos dentro de las ciencias sociales orientadas al estudio del campo religioso en las últimas décadas (Parker, 1996; Deiros, 1997; Fediakova, 2013; Panotto, 2014). Los estudios de la relación entre evangélicos y espacio público varían según las orientaciones en lo referido a la definición de la particularidad del sector como a su relación con lo estrictamente sociopolítico: encontramos enfoques sobre grupos específicos que componen el campo (como los extensos trabajos sobre el pentecostalismo: Frigerio, 1994; Álvarez, 1995; Semán, 2000; Anderson, 2007), definiciones sobre los tipos de campo de incidencia (Carozzi, 1993; Carbonelli, 2008; Mansilla, Orellana y Panotto, 2019) o según maneras de definir el enmarque general a partir del cual se entiende la relación evangélicos-política (Panotto 2015a, 2015b).

Sin caer en reduccionismos analíticos, se podría decir que el estudio de la incidencia evangélica en el espacio público dentro del Cono Sur ha ido a la par de las transformaciones de la presencia política de dichos grupos en América Latina. Encontramos corrientes con énfasis en los siguientes campos: (i) el impacto del crecimiento demográfico a partir de la década de 1950 (Deiros, 1997); (ii) las primeras incursiones e intentos de conformación de partidos confesionales (Deiros, 1987); (iii) el lugar de las comunidades evangélicas en las transformaciones dentro de los sectores populares a partir de la década de 1990 en la coyuntura neoliberal de la región (Míguez, 2000; Semán y Míguez, 2006; Algranti, 2006); (iv) y los nuevos procesos de articulación regional de movimientos evangélicos a partir de principios del 2000 (Carbonelli, 2011).

Las investigaciones mencionadas se han concentrado más específicamente en el análisis de los dos primeros ejes señalados en el inicio; a saber, las dimensiones microsociales y comunitarias del impacto que generan el trabajo de iglesias locales y experiencias individuales de creyentes, así como la incidencia dentro de una matriz nacional-estatal, encauzada en la visibilización y movilización de algunos sectores de iglesias evangélicas. Sin embargo, hay muy pocos estudios desde una mirada más bien regional y desde una 
dimensión política multilateral-internacional del campo (Haynes, 2001; Haddad, Smith y Espósito, 2003; Anderson, 2007; Contins, 2008; Synan, Yong, Álvarez, 2014).

Dado que la gran cantidad de investigaciones sobre el campo evangélico latinoamericano son realizadas a nivel nacional -tanto desde la historia como la sociología y antropología-, hoy por hoy, teniendo en cuenta la influencia del contexto global, donde los fenómenos religiosos y políticos se hacen más intricados, existe, en consecuencia, una gran urgencia de investigaciones con una mayor complejización y profundización en: (i) un análisis de las nuevas dinámicas de incidencia dentro del espacio público global; (ii) los tipos de articulación de estos sectores con agentes sociopolíticos emergentes en la sociedad civil y la política institucional (especialmente transnacional); y (iii) una comprensión sobre la actuación de grupos políticos formales dentro del sistema interamericano.

El análisis de las dinámicas transfronterizas del campo evangélico está en sintonía con los últimos debates en torno a la teoría crítica del espacio público. Uno de los más conocidos es el de Nancy Fraser, quien desarrolla la idea de espacios públicos transnacionales (Fraser, 2008). Esta autora parte desde una crítica a Jürgen Habermas y lo que define como el marco westfaliano de su noción de lo público, es decir, coextensiva al Estado-nación, enmarcada en un territorio soberano y delimitada a una comunidad política específica, definida desde un conjunto de normas esencializadas de pertenencia. Fraser plantea que lo público requiere ser completamente redefinido desde la "constelación posnacional", donde las soberanías son dispersas, el sentido de pertenencia ya no es ni territorial ni nacional, donde las economías son fluidas y sin fronteras, y los medios de comunicación ya no crean una opinión pública territorial sino transfronteriza. De esta manera, lo público ya no representa simplemente un territorio habitado por normas institucionalizadas y sujetos nominados desde fronteras de identidad homogéneas (como la nacionalidad), sino un espacio de conflicto y disputa a partir de lo que en un ensayo anterior Fraser denomina como contrapúblicos subalternos (Fraser, 1992), es decir, grupos que hacen circular contradiscursos e interpretaciones oposicionales frente a las concepciones hegemónicas (o lo que Habermas entiende como el "espacio público burgués"). Estos contrapúblicos no poseen un enmarque ideológico único, sino que pueden oscilar entre posicionamientos anarquistas y sectores religiosos fundamentalistas.

Estos mismos replanteos se hacen patentes en la relación entre lo religioso y lo público. Los fenómenos exógenos de los procesos de globalización y los endógenos vinculados al constante proceso de pluralización de los cuerpos religiosos conlleva a ubicar las dinámicas transfronterizas de la movilidad religiosa no solo como una instancia de transpaso territorial (misional, si se quiere), sino como una nueva matriz de construcción identitaria, en la que los procesos de minoritización de las dinámicas religiosas se ubican como expresiones que compiten con otros agentes hegemónicos por la definición de lo común (Burity, 2015). Como mencionamos anteriormente, lo religioso se transforma en un contrapúblico subalterno que compite y tensiona los significantes circulantes en el espacio público, no necesariamente desprendiéndose de las demarcaciones institucionales, sino reapropiándose de ellas desde mecanismos de singularización, con el propósito de ofrecer sentidos de posición contrahegemónica (Burity, 2018) desde diversos frentes ideológicos posibles.

El presente artículo pretende aportar al área de estudio de la incidencia del campo evangélico desde una clave regional y transfronteriza. Se analiza concretamente dos tipos de configuración de esta incidencia. La primera es denominada como Redes Políticas 
Evangélicas (RPE), que en varios aspectos se acerca a una reactualización de la conocida categoría de religiones públicas de José Casanova.

Las religiones públicas de la sociedad política pudieran ser conceptualizadas como la auto-organización colectiva y la movilización de los grupos religiosos y sus recursos institucionales como grupos de interés que compiten con otros grupos de interés para situar sus ideales e intereses materiales en la arena política. (Casanova, 2012, p. 131)

Dichos grupos, según Casanova, adoptan tres formas principales: movilización de grupos religiosos como movimientos sociales; lobby institucional a nivel local, estatal y federal; y movilización electoral de sectores religiosos y la posible organización en torno a partidos políticos.

En este trabajo entendemos las RPE como grupos que complejizan aún más el análisis de Casanova, principalmente por la proyección transnacional que realizan de los dos primeros puntos recién mencionados. Más concretamente, nos referimos a organizaciones y movimientos vinculados al campo evangélico con las siguientes características: (i) tienen por objetivo trabajar en agendas focalizadas en temáticas políticas (relación con gobiernos y partidos, tratamiento de leyes y políticas públicas, articulación con organizaciones de sociedad civil (OSC), entre otros); (ii) poseen un alcance regional, no solo local o nacional; (iii) están compuestas por personas vinculadas al campo evangélico, pero sin una relación orgánica con un tipo de iglesia, denominación o federación particulares; son más bien espacios integrados por pastores, líderes y profesionales de iglesias evangélicas, de diversas denominaciones e inclusive latitudes, que conforman un espacio de incidencia con una agenda específica en tanto organización política, punto a partir del cual se vinculan con creyentes individuales, iglesias y otras organizaciones basadas en la fe (OBF) que se identifiquen con su propuesta, pero que no necesariamente representan a todo el espectro evangélico.

La segunda configuración son las articulaciones entre RPE. Es decir, el trabajo conjunto que realizan entre diversas redes existentes en América Latina dentro de instancias regionales y organismos multilaterales. En este segundo caso nos referimos concretamente a la presencia de estos grupos en las acciones de la sociedad civil (SC) en espacios como la Organización de Estados Americanos (OEA) y la Comisión Interamericana de Derechos Humanos (CIDH) Por ejemplo, la OEA tiene anualmente su asamblea con miembros de Estado, y en cada ocasión mantiene un diálogo con OSC y actores sociales, previo al encuentro. Inicialmente, no existían tales instancias. Con el tiempo, se crearon mesas temáticas compuestas por organizaciones que se inscribían previamente al encuentro, en las cuales se confeccionaban las vocerías de la SC frente a los representantes de Estado para cada una de las temáticas. A partir de 2017, debido a las dificultades y limitaciones de la práctica de diálogo por mesas temáticas, se cambió el sistema por uno asambleario, con una configuración muy similar al encuentro formal anual del organismo, donde participan representantes de Estado y de las OSC a través de coaliciones, compuestas por un mínimo de diez organizaciones miembros que se aglutinan alrededor de una temática particular.

Para el Diálogo con la Sociedad Civil, son alrededor de 25 coaliciones las que finalmente se escogen para participar en las actividades el día previo a las sesiones formales de la asamblea anual de la OEA. En dichos diálogos asiste la mayoría de los representantes del Estado frente al organismo, los cuales escuchan las peticiones de cada una de las coaliciones en torno a diversas temáticas, que van desde reclamos territoriales hasta la 
construcción de políticas públicas para la comunidad LGBTIQ o el bloqueo de cualquier proyecto de ley sobre despenalización y legalización del aborto en nombre de la "familia natural". 1

Desde el comienzo de este sistema de participación, grupos evangélicos han intervenido activamente a través de coaliciones específicas. Por ejemplo, en la asamblea en Washington de 2018 se inscribieron tres: la Coalición Congreso Evangélico Iberoamericano, la Coalición Brasileira y la Coalición Educación y Cultura por la Democracia. En 2019, de las diez coaliciones identificadas como "profamilia" (de un total de 33), cinco pertenecían al mundo evangélico: Coalición para el Progreso de la Sociedad, Coalición Construyendo Nuevos Horizontes, Coalición Congreso Evangélico Iberoamericano, Coalición Educación y Cultura para la Democracia, y la Coalición Oportunidades para el Ordenamiento Social. En total, estas últimas cinco coaliciones aglutinan un conjunto de 94 organizaciones de sociedad civil e iglesias. Detrás de esta configuración, existe un movimiento regional llamado Congreso Iberoamericano por la Vida y la Familia -fundado en México en el año 2017, que nuclea a iglesias y organizaciones de 17 países, en su mayoría latinoamericanos-, y que actúa como paraguas de todas estas coaliciones.

En este trabajo nos proponemos analizar estos nuevos cuadrantes de participación pública de grupos evangélicos neoconservadores, ${ }^{2}$ especialmente desde los dos formatos descritos anteriormente. Para ello, estudiaremos una de las principales redes latinoamericanas existentes, el Congreso Iberoamericano por la Vida y la Familia, instancia en la que veremos cómo una organización nacional adquiere la capacidad de transformarse en una red regional, a partir de la cual coordina distintas acciones dentro del sistema interamericano. De aquí nos adentraremos en el estudio de las distintas coaliciones evangélicas dentro del Diálogo con la Sociedad Civil de la OEA, enfocándonos en un análisis más bien discursivo de sus intervenciones en las asambleas de la OEA 2018 y 2019. ${ }^{3}$ Previamente, nos introduciremos en dos temáticas que creemos fundamentales como marco general para esta discusión: los debates sobre el estudio del fenómeno religioso desde las relaciones internacionales (RRII) y la necesidad de nuevas categorizaciones sobre los tipos de relación entre campo religioso/evangélico y espacio público, con el propósito de delimitar cómo las vinculaciones cobran distintos matices según el tipo de institucionalidad que se analice.

\footnotetext{
${ }^{1}$ Para más información sobre el sistema de coaliciones, ver el documento "El modelo de coaliciones y su impacto en la incidencia de las organizaciones de la sociedad civil en las cumbres y asambleas de la OEA" (Red Latinoamericana por la Democracia, 2018).

${ }^{2}$ Con neoconservadurismo nos referimos a sectores evangélicos que poseen una movilización pública explícita y focalizada en temáticas relacionadas con la denominada "agenda valórica", es decir, relacionados con la militancia por temáticas dentro del campo de la educación sexual, políticas públicas con enfoque de género y salud sexual y reproductiva, y que poseen un trabajo político intencional a través del lobby, vínculo con partidos y otras organizaciones sociales, desarrollo de campañas públicas y en medios de comunicación. Como categoría analítica, se diferencia de grupos conservadores (que responden a la misma agenda valórica, pero que responden a una dinámica más bien intracomunitaria, sin mucha visibilización pública o política) y de grupos fundamentalistas (que poseen un posicionamiento más reactivo y desde una dinámica más radicalizada, lo cual dificulta un trabajo político articulado con otros sectores y grupos de la sociedad).

${ }^{3}$ Valga indicar que la información sobre el funcionamiento de las coaliciones en el Diálogo con la Sociedad Civil de la OEA proviene del trabajo de campo realizado por el autor en las asambleas de 2018, 2019 y 2020, a través de su participación en una de las coaliciones: la Coalición Religiones, Creencias y Espiritualidades en Diálogo con la Sociedad Civil, además de su rol dentro de la mesa coordinadora de poblaciones de sociedad civil dentro del proyecto PASCA (Participación de la Sociedad Civil en las Cumbres de las Américas), dirigido por la Red Latinoamericana por la Democracia (REDLAD).
} 


\section{Lo religioso, lo político, lo secular: contextualizar tensiones en el sistema internacional}

La actuación de sectores evangélicos en el sistema interamericano debe mirarse desde un contexto más amplio de estudio en torno a la creciente participación de grupos religiosos en el sistema internacional (SI). Las discusiones entre especialistas dentro de las RRII no se han enfocado tanto en el lugar o no de lo religioso dentro del campo de la deliberación multilateral (ya que eso se da por sentado), sino en cómo se entiende su presencia o abordaje (Toft, Philpott y Shah, 2011). En este sentido, hay quienes sostienen que en el campo de las RRII -así como en muchos otros- predomina una tensión entre abordajes esencialistas o institucionalistas, en la que lo religioso se define más desde la creencia individual o a partir de los entramados institucionales en los procesos de identificación con fronteras -rituales, doctrinales, simbólicas- homogéneas (Hurd, 2008), y abordajes ético-positivos (Mahmood, 2006) o relacional-dialogales (Wilson, 2012), que entienden lo religioso más bien como procesos complejos y fluidos de identificación, con diversos niveles de acción -vida cotidiana, moral, prácticas políticas, relaciones sociales, entre otras-, que se construyen a partir de un entramado de diversos tipos de institucionalización (individual, grupal, político, eclesial, o desde configuraciones más amplias, como federaciones religiosas, organizaciones basadas en la fe o redes políticas de incidencia transnacional), que no necesariamente se representan o relacionan con los discursos y formas hegemónicas establecidas por las instituciones oficiales; más bien, emergen como prácticas alternativas y heterodoxas. Dependiendo del punto de partida de la comprensión y definición de lo religioso serán los tipos de abordajes con campos como las políticas públicas y los diálogos multilaterales (Asad, 2003).

Elizabeth S. Hurd sostiene que en el SI -especialmente en las RRII- predomina un concepto institucional, político y jurídico en torno a lo religioso, en el que las creencias se transforman en objetos reificados, con fronteras clausuradas y sin relación con otros campos de lo social. Por ello, el análisis religioso dentro del SI se focaliza más bien en perspectivas cristiano-céntricas (es decir, no solo sobre grupos cristianos concretos, sino desde una metodología de análisis que se construye desde las matrices judeocristianas y su lugar en la narrativa política moderna, matrices que son aplicadas a todas las expresiones religiosas por igual), monoteístas (vale decir, desde la mirada institucional de las religiones mayoritarias y no desde las minorías o expresiones heterodoxas dentro de dichas religiones) y desde una impronta político-jurídica (orientada a marcos institucionales y no tanto en la pluralidad de procesos socioculturales).

Esto marca la preeminencia de concepciones que dejan fuera otro tipo de definiciones de lo religioso (menos institucionales y más socioculturales) y expresiones religiosas específicas en los márgenes de las estructuras oficiales, y con ello otros modos de comprensión sobre la vinculación "oficial” entre Estado, religiones y espacio público. De aquí, afirma Hurd, que esta carencia de visiones más complejizadas y plurales en los discursos de los organismos oficiales dentro del SI sobre libertad religiosa, sobre el reconocimiento de las "minorías", y el reconocimiento de las tensiones y reapropiaciones que se producen desde la religiosidad en la vida cotidiana, paradójicamente, produce una legitimación de miradas y prácticas acotadas que fomentan la exotización de grupos disidentes o no oficiales, derivando finalmente en miradas discriminatorias y hasta violentas (Hurd, 2011). 
Según Hurd (2004), este tipo de distinciones en el campo de las ciencias sociales ha traído tres consecuencias: la necesidad de identificar métodos y teorías sobre la relación creencia-secularización siempre se ha inclinado hacia el lado "secular" del binomio, por representar la faceta "racional"; una tendencia a estudiar lo secular no en relación a lo religioso, sino tomando este último como un objeto independiente que suele "infiltrarse" en el campo de la política; que definiciones monoteístas de lo religioso sean tomadas como normativas.

Es por ello que los espacios multilaterales se transforman en instancias donde emergen tensiones entre las visiones jurídicas e institucionales de lo religioso y la política, y las dinámicas marginales de las "religiones vividas" (Hurd, 2011; Rabia, Morello, Da Costa y Romero, 2019). En este contexto, nacen distintas narrativas sobre cómo lidiar con dichas tensiones. Según Hurd, predominan dos: las lógicas separatistas (mediante las cuales se promueve una división tajante entre lo religioso y lo político, la creencia y la secularización) y las visiones restaurativas (que apelan a un "regreso" de lo religioso, pero demarcando estrictas fronteras entre un campo y otro). Ambas perspectivas, al final, enfatizan una sobredemarcación de jurisdicciones entre campos.

Por todo esto, Hurd advierte sobre los peligros que puede llegar a tener, en términos políticos, una definición acotada de lo religioso. Para ella:

La estabilización de la 'religión' como objeto de gobernanza legal y política global no es una salida desde la presunción secularista, como muchos defensores de la narrativa restaurativa afirman. Más bien, esto re-instala y energiza divisiones particulares entre religión-religión y religión-secular dadas por sentado en la organización de principios públicos de la vida internacional. En lugar de re-acomodar lo religioso, la narrativa restaurativa reforma y cercena binarismos oposicionales específicos entre religión-secular, haciendo ilegibles un conjunto de formas políticas y religiosas disidentes. (Hurd, 2017, p. 98)

De ahí que este autor proponga una lógica de la desecularización, que permita salirnos de los binomios dominantes, para avanzar hacia una visión complejizada y situada de la relación entre estos complejos cambios. Es lo que Hurd denomina como secularización agonística,

donde la 'libertad' emerja como un sitio transitorio de resistencia o modo de insurrección, en lugar de una forma religiosa o política de disciplina impuesta por las autoridades. En lugar de reforzar algo desde arriba, esto está ligado precisamente a lo que las autoridades definen como lo otro, lo no ortodoxo, la disidencia." (Hurd, 2013, p. 235)

En términos operativos, esto significa que la relación religión-política-secularización debe ser contextualizada, historizada y politizada a partir de los diversos modos de comprensión de la relación entre la política, los distintos modos de vivencia religiosa y su relación con otros campos de la vida social, así como los innumerables tipos de articulación entre agentes sociopolíticos y religiosos (Hurd, 2017), dentro de los cambiantes escenarios históricos en los cuales dichos procesos se adscriben, y comprendiendo que tanto "lo religioso" como "lo secular" son significantes que circulan y adquieren una configuración específica a partir de los dispositivos discursivos, contextuales, institucionales y culturales que entren en juego. Lo mismo con la relación entre lo religioso y lo secular (Hurd, 2011), 
lo cual permite tensionar formas particulares de la relación religión-religión y religiónsecular. De ahí que el elemento "internacionalista", intercultural o global del análisis religioso sirve al entrecruzamiento de múltiples variables, contextos y situaciones, donde la relación religión-política puede mostrar tendencias, objetivos y consecuencias muy versátiles, y cuya complejidad y labilidad pueden proyectarse más allá de un análisis de encuadre exclusivamente nacional o desde una perspectiva únicamente jurídica o institucional.

El abordaje de Hurd apunta más bien a cómo situar la idea de pluralismo religioso en el ámbito de las RRII, a cuestionar los conceptos coloniales y legales de lo religioso que predominan en el SI desde una mirada occidental y moderna, y a criticar el lugar de los agentes, dinámicas y discursos hegemónicos dentro de estos escenarios. Aunque no es la intención de la autora, en este trabajo creemos que su propuesta puede servirnos para el análisis de las dinámicas neoconservadoras en las RPE por dos motivos. Primero, porque el aumento y empoderamiento de ciertos discursos neoconservadores dentro del SI son precisamente resultado de las carencias, reduccionismos y falta de precisión sobre la comprensión del mundo religioso por parte de los diversos agentes que componen estos espacios, desde los Estados hasta las OSC. La hegemonía de narrativas y dinámicas religiosas institucionales dentro del campo de las RRII ha llevado a no advertir la creciente incidencia de sectores religiosos disidentes dentro del espectro neoconservador en la región.

En segundo lugar, porque la especificidad identitaria que imprime la presencia de estas redes se presenta muchas veces como "disidente", es decir, como instancias marginales y por fuera de las lógicas institucionales religiosas y políticas más relevantes dentro del juego político internacional. Esto responde en buena medida a lo que Joanildo Burity denomina como procesos de minoritización (Burity, 2016, 2017) o lo que Juan Marco Vaggione (2005) denomina como política reactiva, en la que una expresión generalmente en los márgenes se levanta desde una particularidad (minoritaria) para ejercer presión en términos tanto sociopolíticos como religiosos frente a los discursos, prácticas y modos de institucionalización hegemónicas, en este caso, en oposición a los discursos de organizaciones de derechos humanos dentro del SI. Es decir que la emergencia de estas redes responde, en alguna medida, a un contexto de cuestionamientos y crisis tanto políticas como religiosas, como por ejemplo del lugar social de la Iglesia católica, los movimientos sociales, las OSC y las políticas de derechos humanos (DDHH) promovidas por algunos Estados de la región.

Por eso es importante la propuesta de Hurd en torno a la relevancia de definir e identificar los tipos de inscripción religión-política desde una ubicación precisa, contextualizada e historizada. En este sentido, ¿qué tipo de especificidad promueven las RPE? ¿A qué sentidos de lo político reaccionan y qué cosmovisiones proponen? ¿Cuáles son las narrativas y cosmovisiones hegemónicas que disputan? ¿Frente a quiénes reaccionan y con quiénes se articulan? Estos tipos de vinculación demarcarán fronteras con impacto tanto hacia dentro de las redes como en el campo donde se mueven.

\section{Sobre la necesidad de nuevas categorizaciones en torno a la incidencia evangélica}

La delimitación, contextualización y ubicación con mayor rigurosidad del lugar de los grupos evangélicos que queremos analizar, nos lleva a un interrogante fundamental: ¿cuán representativas son estas redes de "la iglesia evangélica latinoamericana"? En otros términos: más allá de la identificación con el mundo evangélico como marco identitario, ¿hasta qué 
punto dichos grupos tienen una conexión con sectores eclesiales "oficiales”? ¿Pueden ser tomados como instancias representativas de todo el bloque evangélico regional?, ¿desde dónde?, ¿cuál es su objetivo? ¿Presentan dinámicas distintivas si los ubicamos en un encuadre más amplio en relación con el trabajo de las iglesias y las denominaciones, en espacios comunitarios o nacionales?

Los sectores evangélicos que operan dentro del sistema interamericano merecen ser inscritos con mayor especificidad en términos de su definición política y demarcación institucional, evitando hacer una comparación superficial o directa con el campo evangélico como un todo. Tanto las iglesias evangélicas como el propio mundo de la SC son sumamente complejos y diversos, por lo que sería analíticamente incorrecto establecer un vínculo estrecho entre estas redes y las iglesias evangélicas como conjunto. Aquí creemos fundamental aplicar la propuesta de Hurd sobre la contextualización e historización de estas redes, desde su especificidad identitaria y modus operandi, con lo religioso, lo evangélico y lo político, como grandes significantes que son reapropiados de formas específicas, creando con ello distintos modos de diputa de sentido entre religión-política y religión-religión.

$\mathrm{Si}$ analizamos los tipos de visibilización y los espacios en que dichas redes y movimientos trabajan, podemos ver que las agendas, los discursos y los proyectos comienzan a acotarse más taxativamente, inscribiéndose en una polarización más estrecha y representativa de las tensiones del mundo propiamente político-institucional y de la misma sociedad civil, según cada caso y configuración institucional. Por ello, es importante identificar cómo las polarizaciones específicas que se conforman en el mundo de las OSC impulsan polarizaciones específicas (pero muy diversas) en el mundo evangélico como un todo, pero de manera más particularizada y polarizada cuando hablamos de las RPE como institucionalizaciones específicas.

En otros términos, los grupos evangélicos que trabajan en el sistema interamericano deben ser contextualizados, historizados y politizados en un escenario específico, que dé cuenta de las dinámicas del campo donde operan; a saber, las OSC y el modo en que el SI aborda lo religioso en sus distintos campos institucionales y de diálogo multilateral. Podemos decir, sin caer en reduccionismos, que en el campo del Diálogo con la Sociedad Civil en la OEA predominan dos grandes "bandos" (reconocidos extraoficialmente dentro del conjunto de coaliciones, en diversas instancias de encuentro que el organismo ha establecido para los espacios en el proceso previo al Diálogo): uno que se autodenomina conservador (donde se articulan grupos provida, críticos con las agendas feministas y que apuntan a un discurso de defensa de la soberanía nacional, en contra de toda intervención "externa" que promueva cambios en las políticas públicas nacionales) y otro vinculado al mundo de los derechos humanos (donde las minorías sociales están representadas). Las RPE y las coaliciones evangélicas se relacionan principalmente con el primero.

Es desde esta especificidad que denominamos a estos grupos redes políticas evangélicas (RPE) para tratar de destacar su especificidad en términos discursivos y estratégicos, la cual se asume a partir de las prácticas y discursos distintivos del mundo de la sociedad civil en el sistema interamericano, y que a su vez presentan divergencias y diferencias con otro tipo de identificaciones evangélicas, como las que pueden ubicarse en el amplio campo de las iglesias locales, los cuerpos denominacionales o las organizaciones basadas en la fe (también reconocidas como OBF). Con RPE nos referimos, entonces, a grupos compuestos mayoritariamente por líderes eclesiales, pastores/as vinculados/as a movimientos evangélicos regionales, y profesionales del mundo político y el campo de la intervención social (académicos/as, técnicos/as en el área de políticas públicas, 
politólogos/as, abogados/as, economistas, entre otros/as), que poseen una filiación con la Iglesia evangélica, pero que no necesariamente representan una expresión eclesial y denominacional única. Son más bien movimientos y redes paraeclesiales que poseen agendas y recursos propios, y que trabajan con distintos sectores del mundo evangélico, así como el político y la sociedad civil. Estas redes tienen objetivos concretamente políticos, tanto a nivel nacional como regional, transfronterizo e internacional: participar de la construcción de proyectos de políticas públicas, hacer presencia en instancias de consulta y deliberación social (a nivel nacional y regional), realizar lobby político, entre otros.

A continuación, ofrecemos un cuadro que nos permite delimitar con mayor precisión los distintos tipos de inscripción política que podríamos identificar entre diversos sectores evangélicos, según su proceso de institucionalización en términos de incidencia política:

\section{Cuadro $\mathbf{N}^{\circ}$ 1. Tipologías de identificación política según configuración religioso- institucional}

\begin{tabular}{lll}
\hline $\begin{array}{l}\text { Tipos de } \\
\text { institucionalización } \\
\text { religiosa }\end{array}$ & Agentes & Tipos de identificación política \\
\hline Comunidades religiosas & Creyentes en general & $\begin{array}{l}\text { Fluido, heterogéneo, antagónico, } \\
\text { diverso, multipolarizado }\end{array}$ \\
\hline $\begin{array}{l}\text { Instancias de liderazgo } \\
\text { intrarreligioso/eclesial/deno } \\
\text { minacional }\end{array}$ & $\begin{array}{l}\text { Líderes internos del cuerpo } \\
\text { religioso particular/cuerpos } \\
\text { pastorales/líderes eclesiales }\end{array}$ & $\begin{array}{l}\text { Defensa frente al privilegio de } \\
\text { otras religiones y manejo de la } \\
\text { homogeneidad identitaria }\end{array}$ \\
\hline $\begin{array}{l}\text { Instancias de articulación de } \\
\text { liderazgo }\end{array}$ & $\begin{array}{l}\text { Articulación entre líderes de } \\
\text { diversas expresiones religiosas }\end{array}$ & $\begin{array}{l}\text { Participación en espacios de } \\
\text { diálogo público, incidencia } \\
\text { interreligioso/eclesial }\end{array}$ \\
& & $\begin{array}{l}\text { intervención la sociedad civil e } \\
\text { protocolares instancias }\end{array}$ \\
\hline $\begin{array}{l}\text { Organizaciones Basadas en } \\
\text { la Fe (OBF) }\end{array}$ & $\begin{array}{l}\text { Instituciones y organizaciones } \\
\text { paraeclesiales }\end{array}$ & $\begin{array}{l}\text { Instancias de intervención } \\
\text { focalizada y articulación con la } \\
\text { sociedad civil }\end{array}$ \\
\hline $\begin{array}{l}\text { Redes Políticas Religiosas } \\
\text { (Redes Política Evangélicas } \\
- \text { RPE) }\end{array}$ & $\begin{array}{l}\text { Grupos de lobby político y } \\
\text { actores dentro del sistema } \\
\text { internacional }\end{array}$ & $\begin{array}{l}\text { Polarización a partir de las } \\
\text { tensiones dentro de sociedad } \\
\text { civil, discusiones sobre políticas } \\
\text { públicas e instancias de diálogo } \\
\text { multilateral }\end{array}$ \\
\hline
\end{tabular}

Fuente: elaboración propia.

Esta delimitación de categorías -que requiere de un desarrollo con mayor profundidad, el cual no podemos realizar en este momento- nos sirve para ubicar, tal como propone Hurd, el contexto y la especificidad en términos de la relación religión-religión y religión-política, en este caso vinculado a las dinámicas particulares del sistema interamericano y las RPE. Esto nos permite enfatizar en el factor de que dichas redes no responden necesariamente al imaginario completo del mundo evangélico, ya que este es sumamente diverso y, aunque en algunos temas predomina un mayor consenso (sobre todo en lo referente a la agenda valórica), es imposible hacer una correlación entre las agendas políticas de estas redes y los grupos evangélicos como un todo. Es otros términos, analizar las características de las RPE implica identificar y desarrollar elementos distintivos de dichas 
redes, muchos de los cuales tienen relación con el espectro evangélico regional, pero que son reapropiados y utilizados de forma específica, dentro del campo del SI.

Finalmente, este esquema posibilita identificar y delimitar con mayor precisión las agendas, los actores, los objetivos y las articulaciones de estas redes, ubicándolas en un contexto y proceso institucional delimitados. De esta manera, podemos encontrar que dichas redes no solo tienen un lugar fundamental en la negociación y tensión de las fronteras de las OSC en el campo del sistema interamericano, sino también provocan instancias de litigio hermenéutico y político en relación con el campo religioso que representan, el cual no es homogéneo y demarca resistencias respecto de muchas de sus agendas. Por ello, más allá del innegable vínculo entre estas redes y la cosmovisión evangélica regional, es también prudente establecer ciertas distancias entre los objetivos de cada campo, para no caer en generalizaciones.

\section{Redes Políticas Evangélicas (RPE) en el sistema interamericano: el caso del Congreso Iberoamericano por la Vida y la Familia}

Una de las características que se destacan de la especificidad de estas RPE es la relación entre las configuraciones más domésticas, locales y propiamente identitarias de expresiones evangélicas, y su mutación hacia procesos más complejos, organizados y con un alcance más extenso, como es el regional. Es decir, el desarrollo y la eficacia demográfica que alcanzan estas redes tiene directa relación con la extensión que obtuvieron de la relación con iglesias evangélicas en distintos países, lo cual les ha otorgado no solo recursos humanos, financieros e institucionales, sino un gran capital simbólico, los cuales han podido proyectar desde una matriz más nacional hacia una regional o global.

Esto lo podemos ver en las características que configuran el surgimiento del Congreso Iberoamericano por la Vida y la Familia, así como su actuación en el sistema de coaliciones de la OEA. Este congreso se compone de una red de iglesias y organizaciones de la cual nacen todas las coaliciones evangélicas que participan en el Diálogo con la Sociedad Civil de este organismo. El andamiaje o imaginario evangélico se expresa desde el nacimiento de este espacio. En su origen, vemos la centralidad de la figura de Aarón Lara Sánchez, pastor y periodista, personaje mediático reconocido en México por ser uno de los fundadores de la Asociación Cristiana de Periodismo (ACP) y la Confraternidad Evangélica de México (CONEMEX). Desde fines de la década de 1990 se hizo conocido por ser el autor de una serie de libros y estudios sobre temas de género, familia y sexualidad. En 2011 fundó Red Soluciones, una organización orientada a la consultoría para la apertura de organizaciones civiles, especialmente desde iglesias locales. Desde su nacimiento, ayudó a la apertura de 120 organizaciones civiles en todo México. En 2016, Sánchez fundó también Iniciativa Ciudadana por la Vida y la Familia, la cual cobra visibilidad en México cuando el expresidente Peña Nieto intentó poner en discusión una ley de matrimonio igualitario. Esta organización, gracias a su extensa red de organizaciones aliadas e iglesias, logró juntar más de 360 mil firmas con el propósito de frenar dicho proyecto.

Siguiendo el impacto social que produjo dicho acontecimiento, y teniendo en cuenta situaciones similares que comenzaron a vivirse en otros países de la región, dicha organización tomó la iniciativa para desarrollar un congreso en México en el año 2017, para el cual se convocó a representantes de 17 países (Argentina, Bolivia, Chile, Colombia, Costa Rica, Ecuador, España, Guatemala, Honduras, México, Nicaragua, Panamá, Paraguay, Perú, Puerto Rico, República Dominicana y Uruguay), con el propósito de conformar un frente 
entre iglesias y organizaciones dentro del espectro evangélico, para posicionarse frente a lo que identificaban como la escalada de la "ideología de género" en la región. En este evento se firmó la "Declaración de Santa Fe", cuyas temáticas centrales fueron: la promoción de la familia como núcleo de la sociedad, una crítica a las políticas públicas sobre género y la defensa de la vida desde la concepción, argumento desde el cual se oponen a cualquier tipo de legislación a favor del aborto.

A partir de este evento, la organización Iniciativa se bifurca entre un cuerpo con énfasis local y otro regional: por un lado, nace el Congreso Iberoamericano por la Vida y la Familia como una red internacional, mientras el anclaje en México queda bajo Iniciativa Ciudadana, una ONG que actúa en todo el país a través de la convocatoria de movilizaciones en distintas ciudades, la articulación con organizaciones civiles para el tratamiento de agendas comunes y el desarrollo de un fuerte lobby político entre diputados, senadores, gobernadores y ministros de distintas áreas.

En este Congreso y su declaración final, denominada "Declaración de Santa Fe", no solo se estipulan puntos de partida doctrinales, morales o ideológicos, sino que se establece una clara agenda política, a través de cinco puntos: se hace un llamado a la construcción de:

1.- Mecanismos de coordinación con otras entidades iberoamericanas que comparten objetivos. 2.- Puesta en marcha de un Centro de Estudios. 3.- Preparación de coaliciones de ONGs para lograr presencia y voz en la próxima asamblea de la OEA, y en otras organizaciones internacionales. 4.- El lanzamiento de un medio evangélico para Latinoamérica en formato digital, y 5.- La conformación de una Fraternidad de Parlamentarios Evangélicos. (Congreso Iberoamericano por la Vida y la Familia, 5 de junio de 2017, s.p.)

En el año 2018 se llevó a cabo un segundo congreso en México, en febrero de 2019 otro en Panamá y se desarrolló un último encuentro durante marzo de 2020 en Perú. El anclaje local no solo queda en manos de Iniciativa Ciudadana, sino también de Concertación AC, Centro de Cultura y Orientación Civil, el cual actúa bajo la configuración de un think tank a través del cual se coordinan instancias de comunicación política y un programa de formación para jóvenes, llamado BECARIOS. Este Congreso - el cual trabaja con otras redes regionales como la Alianza Evangélica Latina (AEL), "Con Mis Hijos no te Metas", Parlamento y Fe, entre otros-, logra incidir en el Diálogo de la Sociedad Civil mediante la conformación de estas cinco coaliciones, las cuales se articulan con otras coaliciones aliadas en agendas y reclamos comunes.

En resumen, el nacimiento y la performance del Congreso en el marco de la OEA nos permite ver varios de los elementos fundamentales sobre la construcción de nuevas lógicas de incidencia: (i) el desarrollo de una red de alcance regional apoyada en el establecimiento de una plataforma nacional, desde representaciones eclesiales y de organizaciones basadas en la fe; (ii) un proceso de articulación con diversos actores de la sociedad civil para la construcción de instancias de incidencia, presión, lobby y disputa de sentido discursivo; (iii) la evidencia de polarizaciones que caracterizan a la sociedad civil latinoamericana; (iv) la construcción de una estrategia política regional con el objetivo de lograr instancias de incidencia a nivel local y nacional. 


\section{RPE y el sistema de coaliciones en el sistema interamericano}

Como hemos indicado, la presencia evangélica en el sistema interamericano se hizo más notoria a partir de la participación del Diálogo de la Sociedad Civil en la OEA a través de la conformación de diversas coaliciones. Cabe indicar que más allá de que existen coaliciones explícitamente evangélicas, hay otras que se inscriben con nombres genéricos, sin presentar una identificación religiosa y a partir de organizaciones sociales independientes, pero que poseen una vinculación con diversos grupos evangélicos o católicos. En este apartado nos concentraremos en identificar algunos de los puntos más importantes en los discursos realizados por estas coaliciones en las instancias de Diálogo con la Sociedad Civil en la $48^{\mathrm{a}}$ y $49^{\mathrm{a}}$ asamblea de la OEA. ${ }^{4}$

La centralidad sobre los temas alrededor de la llamada "agenda valórica" constituye la columna vertebral de la demanda de estas coaliciones. El cuestionamiento a legislaciones sobre la diversidad sexual, la negación del derecho al aborto, la crítica a los movimientos feministas y LGBTIQ, todo esto basado en la defensa de la "familia natural" y de los "valores fundamentales". La particularidad que se destaca de los discursos sobre estos temas es la vinculación que se hace entre esta agenda valórica y el desarrollo sociopolítico y económico de los países. Es decir, la defensa de los valores tradicionales, según estas coaliciones, tiene directa relación con el desarrollo socioeconómico, la estabilidad social y la construcción de un ambiente democrático. Su negación o la promoción de otros temas y agendas contrapuestas significan poner en peligro la estabilidad en cada uno de esos ámbitos. En varios casos, además, dicha perspectiva se cimenta sobre el supuesto fundamento científico que poseen las aseveraciones a los valores y principios esgrimidos.

[...] para hablar de un cáncer establecido en tantas naciones americanas que ha frenado el desarrollo y la justicia social de las mismas. Es el motivo de la paralización de mi nación y muchas naciones de las Américas: la corrupción. Sin embargo, tenemos que alertar, que hay dos "Cánceres" que comienzan a tomar proporciones mayores y que van a traer una crisis mucho mayor, no sólo a mi nación pero las demás: el cáncer de la devaluación de la vida y la familia. Son dos bases que afectarán estructuralmente a las naciones [...] Estamos a favor del desarrollo de todas las naciones para que tengamos justicia social, pero no creemos en el desarrollo sin la valorización del derecho a la vida ya la familia. (Congreso Iberoamericano por la Vida y la Familia, 5 de junio de 2018a, s.p. $)^{5}$

No hay progreso con familias desintegradas y con niños a quienes se les hace dudar de su identidad. La cohesión es un valor de futuro para nuestro continente. (Congreso Iberoamericano por la Vida y la Familia, 8 de julio de 2019a, s.p. $)^{6}$

Un discurso muy recurrente en varias de las coaliciones -no solo de las evangélicases la defensa de la soberanía nacional y el cuestionamiento hacia cualquiera "intromisión externa", incluyendo a la propia OEA y el CIDH. Este recurso se eleva con el propósito de

\footnotetext{
${ }^{4}$ Las fuentes de referencia, además de la presencia del autor en ambas actividades como observador, fueron extraídas del sitio oficial de la OEA (2018, 2019). Además, se utilizó el canal de YouTube del Congreso Iberoamericano por la Vida y la Familia, disponible en: https://www.youtube.com/channel/UCMqPV2uig2qOVzF-itfPWeA

${ }^{5}$ Coalición Brasileira.

${ }^{6}$ Coalición Construyendo Nuevos Horizontes.
} 
evitar y cuestionar cualquier denuncia por violaciones de derechos humanos en algún país o la promoción de proyectos de ley que vulneren o pongan en tensión las fronteras constitucionales de una nación. Este mismo mecanismo es el que están utilizando coaliciones evangélicas para criticar las posibles "intromisiones ideológicas" en y desde los Estados, especialmente en el campo educativo y valórico.

En esta línea, el Estado debe asegurar los recursos básicos para el desarrollo integral de las personas, pero debe renunciar a una actitud de tutelaje integral; el Estado no debe tener ideología y no debe imponer ninguna agenda concreta a la sociedad. Esto es especialmente importante en un área fundamental para el desarrollo de la sociedad como es la educación: el estado debe reconocer el área de competencia de los padres y no competir con ellos, sino respetar su área de soberanía y colaborar con ellos en la transmisión e integración de valores solidarios [...] Principal factor de descomposición social en nuestro continente: el descolocamiento de la familia natural. Los problemas sociales se solucionan con 'familias naturales fuertes'. (Congreso Iberoamericano por la Vida y la Familia, 8 de julio de 2019b, s.p.) ${ }^{7}$

Esto último también se vincula con una fuerte delimitación con respecto a otros grupos y sus demandas, más concretamente presentando las propuestas de OSC relacionadas con DDHH como una amenaza a los fundamentos de la convivencia democrática (Panotto 2019a). Por esta razón, otro elemento muy fuerte en estos grupos es lo que podríamos denominar como un llamado a la desideologización de la política. Aunque no utilizan con tanta frecuencia el concepto de "ideología de género", realizan aseveraciones desde los mismos objetivos retóricos y políticos que dicha expresión representa. Es decir, deslegitiman la participación o demanda de otros sectores desde la denuncia de "ideologización" del reclamo, planteando su propuesta desde un estatus de objetividad otorgado por la ciencia, el sentido común, la razón o la extensión de procesos históricos (Panotto, 2019b). También se pueden ver reclamos sobre supuestas campañas conspirativas e internacionales como intentos de intervención o incidencia en planos globales.

Pedimos por ejemplo, que esta representación internacional se abstenga de atentar contra el idioma español al incluir un lenguaje que pretende ser inclusivo yendo en contra de lo dispuesto por la RAE. (Congreso Iberoamericano por la Vida y la Familia, 8 de julio de 2019a, s.p. $)^{8}$

A pesar de esta denuncia sobre la "ideologización" de ciertos discursos, es sugestivo ver cómo se evidencia en estos sectores una reapropiación de discursos dentro de la agenda de los derechos humanos. Hay una apelación al sentido de libertad, igualdad, libertad de expresión, laicidad, independencia, entre otros, pero con un giro retórico que se contrapone al trasfondo político que apelan y se relaciona más directamente con las propuestas particulares en torno a la agenda valórica.

Estas medidas permitirán generar una cultura de respeto e igualdad frente a las libertades civiles como el derecho de expresión, de asociación, de libertad religiosa, de pensamiento, así como una mayor calidad educativa científica y ajena a ideologías

\footnotetext{
${ }^{7}$ Coalición para el Progreso de la Sociedad.

${ }^{8}$ Coalición Construyendo Nuevos Horizontes.
} 
subjetivas, como derecho humano de niñas, niños y adolescentes de la región. (Congreso Iberoamericano por la Vida y la Familia, 5 de junio de 2018b, s.p.) $)^{9}$

En este sentido, la coalición que representamos propugna valores que contrastan con la tradición controladora que ha creado dependencias indeseables y ha lastrado el avance de nuestras naciones; propugnamos así valores de progreso, que dignifican a la persona, promueven su autonomía, sus libertades, su responsabilidad y su conciencia social. (Congreso Iberoamericano por la Vida y la Familia, 5 de julio de 2019a, s.p. $)^{10}$

Desde la independencia, el objetivo ha sido crear una educación laica y científica, liberada de las ataduras dogmáticas. (Congreso Iberoamericano por la Vida y la Familia, 5 de julio de 2019b, s.p. $)^{11}$

Uno de los elementos más importantes a destacar sobre el discurso de estas coaliciones es cómo lo religioso (y lo evangélico en particular) ya no se presenta solo como la expresión de una identidad específica, sino como el establecimiento de elementos que sirven a la construcción de un proyecto político, con repercusión para toda la sociedad. Se asume la identidad religiosa ya no como una disidencia, sino como un agente fundamental para el desarrollo social y con un proyecto político específico. Aquí podemos ver dos tipos de estrategia: una primera es presentar explícitamente el nombre de Dios, la iglesia, lo religioso, como un marco fundacional. Por ejemplo, lo vemos con la siguiente afirmación de la Coalición Brasileira en 2018: "Estamos a favor de la ideología de género: Dios creó al hombre y a la mujer. Esto no es religión, esto es familia" (Congreso Iberoamericano por la Vida y la Familia, 5 de junio de 2018a, s.p.).

Una segunda responde a lo que Juan Marcos Vaggione denomina como secularismo estratégico (Vaggione, 2005), es decir, la apelación a una retórica que se presenta no religiosa, como vía estratégica para transmitir un conjunto de valores religiosos particulares sin que sean cuestionados de sesgo. Es decir, se pretende universalizar un "valor" asociado al campo evangélico, a partir de una retórica política que le otorga una aplicación universal. Uno de los casos más emblemáticos son las intervenciones de la Coalición Congreso Evangélico Iberoamericano. En la asamblea de 2018 interpelaron a los y las presentes a la necesidad de buscar un "enfoque multidimensional", especialmente a la cooperación policial en temas técnicos, científicos y educativos, "mediante la Red Interamericana de Desarrollo y Profesionalización Policial, y la implementación del Acuerdo Marco de Cooperación Interinstitucional suscrito entre la Secretaria general de la OEA y la Comunidad de Policías de América (AMERIPOL)" (Congreso Iberoamericano por la Vida y la Familia, 8 de julio de 2019 c, s.p.). ${ }^{12}$ Esto serviría para "resolver problemáticas que también se relacionan con los derechos humanos más fundamentales", como la proliferación en el continente de lo que llaman "paraísos legales para la subrogación materna o renta de vientres". Ello apunta, según esta Coalición, a desarmar "el tráfico de vientres, a través de la sinergia real y la participación directa e integrada en los cuerpos de inteligencia y cooperación internacional, de civiles

\footnotetext{
${ }^{9}$ Coalición Educación y Cultura para la Democracia.

${ }^{10}$ Coalición Oportunidades para el Ordenamiento Social Contemporáneo.

${ }^{11}$ Coalición Educación y Cultura para la Democracia.

${ }^{12}$ Coalición Congreso Evangélico Iberoamericano [en adelante CCEI]).
} 
expertos en la materia, activistas de derechos humanos" (Congreso Iberoamericano por la Vida y la Familia, 8 de julio de 2019c, s.p.). Esta presentación, que tenía por objetivo dar un enfoque político multilateral sobre una problemática particular, en el fondo pretende más bien destacar la relación entre políticas de despenalización del aborto y el tráfico de bebes.

La intervención en la asamblea de 2019 de esta misma Coalición tuvo una posición mucho más directa en términos de la dimensión política de la identidad evangélica, pero dejando de forma explícita el uso estratégico de cierto lenguaje de derecho liberal. Afirman:

Los evangélicos latinoamericanos somos conscientes de nuestro destacado crecimiento numérico en los últimos años. Este avance ha supuesto cambios sociales en nuestros países y algunos analistas indican que nuestra participación se ha convertido en un elemento crucial en recientes cambios políticos muy relevantes. (Congreso Iberoamericano por la Vida y la Familia, 8 de julio de 2019c, s.p.)

A partir de lo anterior, plantean tener: "un mensaje propio que queremos ofrecer como un instrumento de transformación, progreso social y económico y profundización democrática" (Congreso Iberoamericano por la Vida y la Familia, 8 de julio de 2019c, s.p.), basado en los siguientes puntos: ${ }^{13}$

1. La reafirmación de los derechos de la persona y de las responsabilidades del individuo, que "como evangélicos queremos, desde nuestros códigos éticos, colaborar en su consolidación y desarrollo".

2. El respeto a las minorías y el derecho a la disidencia. "Los evangélicos sabemos lo que es sufrir discriminación y queremos que nuestra experiencia sirva para generar cambios en las mentalidades asentando criterios de tolerancia y respeto a la disidencia".

3. El control del ejercicio del poder. "El modelo de los checks and balances surgió de una mentalidad protestante que entiende que todo poder humano debe ser controlado. Como evangélicos queremos transmitir esta perspectiva con el objetivo de mejorar la salud democrática de nuestros países".

4. La libertad de conciencia y de expresión. "La primera de las libertades es la de conciencia, y como evangélicos tenemos detrás una larga historia de lucha por su conquista."

5. Un concepto claro de separación entre iglesias y estado. "Esto es definitivamente compatible con la aportación de valores religiosos al libre debate de ideas en el diálogo político."

6. "La defensa de la vida humana desde su inicio hasta su fin, y de la familia como elemento fundamental de una sociedad libre; ambos son valores transversales básicos que deben estar por encima de debates ideológicos."

En resumen, esta última intervención pone de manifiesto dos elementos centrales para nuestro análisis. Primero, la profundización en los mecanismos de incidencia e intervención de las RPE a través del uso estratégico de la retórica política no solo como un mecanismo de intervención, sino también de articulación con otros actores y coaliciones. Segundo, se

\footnotetext{
${ }^{13}$ Todas las citas mencionadas a continuación en los siguientes puntos corresponden a Congreso Iberoamericano por la Vida y la Familia (8 de julio de 2019c, s.p.).
} 
evidencia una exposición de la especificidad evangélica, no solo ya como un agente de defensa moral (es decir, como interviniente exclusivamente en temas de agenda valórica), sino como un actor que puede aportar a cambios sociales más profundos y amplios, relacionados con la democracia, las políticas públicas, la libertad de expresión, entre otras. Esto se basa, como vimos, en la exaltación de su crecimiento demográfico. El tema valórico, por su parte, se utiliza como un marco cosmovisional o como una bandera de incidencia, cuyo impacto político alcanza otras áreas de la sociedad y la política.

\section{Conclusiones}

El análisis del accionar de las RPE en el sistema interamericano da cuenta de las propias transformaciones que se están gestando en el SI con respecto al lugar de las religiones. Pero en este caso, no podemos hablar de "religiones" en términos genéricos. Tal como se propuso, hay que comenzar a delimitar qué tipo de actores son los que se movilizan en estas instancias, qué clase de vínculos poseen con las comunidades religiosas que dicen representar y de qué manera se apropian y resignifican políticamente los marcos de sentido que dicen encarnar.

Complejizar estas delimitaciones institucionales y cosmovisionales servirá para ahondar en lo que Elizabeth Sharkman Hurd propone en términos de politización e historización de los múltiples vínculos entre religión-política-secularización, que se juegan dentro de las RRII, con el objetivo de no caer en lecturas reduccionistas o polarizadas. Dar cuenta de estos escenarios significa reconocer el creciente lugar de lo religioso en el escenario de la incidencia regional e internacional, pero desde diversas facetas y expresiones que pueda tomar.

El estudio de las RPE esbozadas en este escrito nos permite ver una mutación de los procesos de minoritización del campo evangélico latinoamericano, desde la configuración de redes de alcance regional, que no pierden las caracterizaciones típicas del imaginario y performance evangélica, pero que construyen nuevos tipos de articulación con las OSC, localizando nuevas disputas de sentido religión-política y religión-religión a través de los siguientes elementos: lo evangélico se presenta ya no únicamente como un marco reactivo, sino más bien propositivo en términos de desarrollo de prácticas sociales y resignificación de sentidos políticos; lo evangélico sirve a la reapropiación de significantes vinculados a la libertad de expresión, los derechos humanos y la democracia, a partir de las múltiples tensiones que se gestan en el campo de las OSC dentro del SI.

Por último, esto nos lleva a entender la incidencia neoconservadora de las RPE desde una perspectiva más extensa, es decir, desde un conjunto delimitado de especificidades políticas, pero a su vez planteando un proyecto político que abarca todas las áreas de la sociedad. De aquí la necesidad de entender la presencia de estos grupos no solo desde la lógica de una "política de shock" o la "politización reactiva" a partir de la promoción de una agenda valórica, sino desde la inscripción de su particularidad -identitaria y discursiva- en un escenario mucho más extenso, donde su figura representa (y se autodefine), en alguna medida, la respuesta frente a ciertos hiatos que se han abierto en los replanteamientos hacia actores sociales, discursos políticos, agendas de políticas públicas y religiosas tradicionales, especialmente en el campo de las políticas regionales. 


\section{Referencias bibliográficas}

Algranti, J. (2006). Notas para el estudio de las comunidades pentecostales. Scripta Ethnologica, (XXVIII), 95-120.

Álvarez, C. (1995). Panorama histórico de los pentecostalismos latinoamericanos y caribeños. En B. Gutiérrez (ed.), La fuerza del espíritu. Los pentecostales en América Latina: un desafío a las iglesias históricas (pp.35-56). México: AIPRAL/CELEP.

Anderson, A. (2007). El pentecostalismo. Madrid: Akal

Asad, T. (1993). Genealogies of Religion. Londres: John Hopkins University Press.

Asad, T. (2003). Formations of the Secular. Christianity, Islam and Modernity. California: Stanford University Press.

Burity, J. (2015). Minoritização, glocalização e política: para uma pequena teoria da translocalização religiosa. Cadernos de Estudos Sociais, 30(2), 31-73.

Burity, J. (2016). Minoritization and pluralization. What is the "people" that pentecostal politicization is building? Latin American Perspectives, 43(3), 116-132.

Burity, J. (2017). Autoridad y lo común en procesos de minoritización. El pentecostalismo brasileño. Revista Latinoamericana de Investigación Crítica, 4(6), 99-125.

Burity, J. (2018). Espiritualidades del consumo, del resentimiento y del agonismo político: religión pública versus desinstitucionalización religiosa. En J. Cruz Esquivel y V. Béliaveau (coords.), Religión en cuestión: campos, fronteras y perspectivas (pp. 283308). Buenos Aires: CICCUS Ediciones/ACSRM.

Carbonelli, M. (2008). Evangélicos y política en Argentina: entre la institucionalización y la autonomía. Mitológicas, 23(1), 47-65.

Carbonelli, M. (2011). Ciencias sociales, evangélicos y política. Una lectura sobre la producción científica acerca de la participación política evangélica en la vida democrática argentina (1983-2010). Revista Cultura y Religión, V(2), 96-116.

Carozzi, M. J. (1993). Tendencias en el estudio de los nuevos movimientos religiosos en América: Los últimos 20 años. Revista Sociedad y Religión, 10/11(1), 10-31.

Casanova, J. (2012). Genealogías de la secularización. Madrid: Anthropos.

Congreso Iberoamericano por la Vida y la Familia (5 de junio de 2017). Declaración de Santa Fe. [Consultado el 15 de enero de 2020]. Recuperado de: http://congresoiberoamericanoporlavidaylafamilia.org/declaracion-de-santa-fe/

Congreso Iberoamericano por la Vida y la Familia (5 de junio de 2018a). Glaucio Coraiola, Brasil. Mensaje ante la 48 Asamblea de la OEA [archivo de video]. [Consultado el 15 de enero de 2020]. Recuperado de: https://www.youtube.com/watch?v=HA9ORKKhbYE\&list=PLBnzhNb6ki1xbBLePj3ZxV0_pRs1OPRb.

Congreso Iberoamericano por la Vida y la Familia (5 de junio de 2018b). Gilberto Rocha, México. Mensaje ante la 48 Asamblea de la OEA [archivo de video]. [Consultado el 15 de enero de 2020]. Recuperado de: https://www.youtube.com/watch?v=Jn8ohiWQV78\&list=PLBnzhNb6ki1 xbBLePj3ZxV0_pRs1OPRb\&index $=2$

Congreso Iberoamericano por la Vida y la Familia (5 de julio de 2019a). Voceros en la OEA: Clara Vega de Rocha [archivo de video]. [Consultado el 15 de enero de 2020]. Recuperado de: https://www.youtube.com/watch? $\mathrm{v}=\mathrm{Wf2}$ sjlZ2Wuk\&t=32s 
Congreso Iberoamericano por la Vida y la Familia (5 de julio de 2019b). Voceros en la OEA: Patricia Cortés [archivo de video]. [Consultado el 15 de enero de 2020]. Recuperado de: https://www.youtube.com/watch? $\mathrm{v}=\mathrm{qg}$ W7Kp9WpIk\&t=12s

Congreso Iberoamericano por la Vida y la Familia (8 de julio de 2019a). Voceros OEA. Silvana Vidal [archivo de video]. [Consultado el 15 de enero de 2020]. Recuperado de: https://www.youtube.com/watch?v=fj3iX3o43nk\&t=11s

Congreso Iberoamericano por la Vida y la Familia (8 de julio de 2019b). Congreso Iberoamericano. Milagros Jáuregui Perú [archivo de video]. [Consultado el 15 de enero de 2020]. Recuperado de: https://www.youtube.com/watch?v=uRJs6Iv8aPo\&list=PLBnzhNb6ki3pbwczGX_iV-T6Kq_B7YPS\&index=5

Congreso Iberoamericano por la Vida y la Familia (8 de julio de 2019c). Voceros OEA 2019 - Marco Camargo. [archivo de video]. [Consultado el 15 de enero de 2020]. Recuperado de: https://www.youtube.com/watch?v=NJihdhqKA1I\&t=2s

Contins, M. S. (2008). Religião, etnicidade e globalização: uma comparação entre grupos religiosos nos contextos brasileiro e norte-americano. Revista de Antropologia, 51(1), 67-106.

Deiros, P. (1987). Los evangélicos y el poder político en América Latina. Buenos Aires: Nueva Creación.

Deiros, P. (1997). Protestantismo en América Latina. Ayer, hoy y mañana. Nashville: Caribe. Fediakova, E. (2013). Evangélicos, política y sociedad en Chile: dejando "el refugio de las masas". 1990-2010. Santiago: CEEP-IDEA-UdeSantiago.

Fraser, N. (1992). Rethinking the public sphere: a contribution to the critique of actually existing democracy. Social Text 25/26(1), 56-80.

Fraser, N. (2008). Escalas de justicia. Barcelona: Herder.

Frigerio, A. (1994). Estudios recientes sobre el pentecostalismo en el Cono Sur: problemas y perspectivas. En A. Frigerio (ed.), El pentecostalismo en Argentina (pp.10-28). Buenos Aires: Centro Editor de América Latina Biblioteca Política Argentina.

Habermas, J. (2009). Historia y crítica de la opinión pública. Buenos Aires: Editorial Gili.

Haddad, Y., Smith, J. y Espósito, J. (2003). Religion and Inmigration. Wilnut Creek: Altamira Press.

Haynes, J. (2001). Transnational religius actors and international politics. Third World Quarterly, 22(2), 143-158.

Hurd, E. S. (2004). Political authority of secularism in international relations. Europan Journal of International Relations, 10(2), 235-262.

Hurd, E. S. (2008). The Politics of Secularism in Internacional Relations. New Jersey: Princeton University Press.

Hurd, E. S. (2011). A suspension of (dis)belief: the secular-religious binary and the study of international relations. En C. Calhoun, M. Juergensmeyer y J. van Antwerpen (eds.), Rethinking Secularism (pp.166-184). Oxford: University Press.

Hurd, E (2013). The international politics of religious freedom. IIC Quarterly, 40(1), 225237.

Hurd, E. S. (2017). Narratives of de-secularizationin in international relations. Intellectual History Review, 27(1), 93-113.

Mahmood, S. (2006). Secularism, hermeneutics, and empire: the politics of islamic reformation. Public Culture, 18(2), 323-347. 
Mansilla, M. y Orellana, L. (2018). Evangélicos y política en Chile 1960-1990. Santiago: UNAP-RILL.

Mansilla, M., Orellana, L. y Panotto, N. (2019). La participación política de los evangélicos en Chile (1999-2017). Revista Rupturas, 9(1), 179-208.

Míguez, D. (2000). Modernidad, posmodernidad y la transformación de la religiosidad de los sectores medios y bajos en América Latina. Revista Ciencias Sociales, 10, 56-68.

OEA (2018). Resumen de presentaciones de las coaliciones de sociedad civil y de los actores sociales. 3 de junio de 2018. Centro de Noticias OEA. [En línea]. Recuperado de: https://www.oas.org/es/centro_noticias/comunicado_prensa.asp?sCodigo=D-028/18

OEA (2019). Resumen de presentaciones de coaliciones de sociedad civil y de actores sociales. 26 de junio de 2019. OEA. [En línea]. Recuperado de: http://www.oas.org/es/49ag/docs/presentaciones-coaliciones/Insumos-de-Coalicion49-Asamblea-General-OEA.pdf.

Panotto, N. (2014). Pentecostalismos y construcción de identidades sociopolíticas. Desafíos 26(2), 73-96.

Panotto, N. (2015a). Pluralismo político y pluralismo religioso: nuevos escenarios y matrices analíticas de la relación. En J. M. Renold (ed.), Religión: estudios antropológicos sobre sus problemáticas (pp. 173-195). Buenos Aires: Editorial Biblos.

Panotto, N. (2015b). Religión, ciudadanía y espacio público: un acercamiento socioantropológico y teológico. Perspectivas Internacionales, 11(1), 63-113.

Panotto, N. (2019a). Incidencia religiosa en la OEA: entre agendas en tensión y denuncias con pobreza democrática. Sitio web de Grupo de Estudios Multidisciplinarios sobre Religión e Incidencia Pública (GEMRIP) Recuperado de: http://www.gemrip.org/incidencia-religiosa-en-la-oea-entre-agendas-en-tension-ydenuncias-con-pobreza-democratica\%EF\%BB\%BF

Panotto, N. (2019b). Fe que se hace pública: reflexiones sobre religión, cultura, sociedade incidencia. Buenos Aires: JuanUno.

Parker, C. (1996). Popular Religion and Modernization in Latin America. Nueva York: Orbis Books, Maryknoll.

Rabia, H., Morello, G., Da Costa, N. y Romero, C. (eds.) (2019). La religión como experiencia cotidiana: creencias, prácticas y narrativas espirituales en Sudamérica. Córdoba: Editorial Universitaria.

Red Latinoamericana por la Democracia (2018). El modelo de coaliciones y su impacto en la incidencia de las organizaciones de la sociedad civil en las cumbres y asambleas de la OEA. Texto inédito.

Semán, P. (2000). El pentecostalismo y la religiosidad de los sectores populares. En M. Svampa (ed.), Desde abajo: La transformación de las identidades sociales (pp.155180). Buenos Aires: Biblos.

Semán, P. y Míguez, D. (2006). Entre santos, cumbias y piqueres. Las culturas populares en la Argentina reciente. Buenos Aires: Biblos.

Synan, W., Yong, A. y Álvarez, M. (eds.) (2014). Global Renewal Christianity. Past, Present and Future. Florida: Charisma House.

Toft, M., Philpott, D. y Shah, T. S. (2011). God's Century: Resurgent Religion and Global Politics. Nueva York: W. W. Norton.

Wilson, E. K. (2012). After Secularism: Rethinking Religion in global Politics. Nueva York: Palgrave Macmillan.

Vaggione, J. M. (2005). Reactive politicization and religious dissidence. The political 
mutations of the religious in social theory and practice. Social Theory and Practice, 31(2), 233-255.

\section{Cómo citar este artículo}

Panotto, N. (2020). Incidencia religiosa en clave multilateral: la presencia de redes políticas evangélicas en las asambleas de la OEA. Revista Cultura \& Religión, 14(1), 100-120. 